\title{
BIOSEGURIDAD EN LA PRESCRIPCIÓN Y TRANSCRIPCIÓN DE TERAPIA MEDICAMENTOSA ENDOVENOSA ${ }^{1}$
}

\author{
PRESCRIPTION AND TRANSCRIPTION BIOSECURITY \\ IN ENDOVENOUS DRUG THERAPY
}

\author{
EDITH RIVAS R. \\ AngÉlica Rivas L**. \\ Luis Bustos M.*
}

\begin{abstract}
RESUMEN
Los errores en el proceso de medicación en sus diferentes fases es un problema que involucra riesgos para el usuario, así como en la calidad de atención de los servicios. Los objetivos fueron: a) Determinar la bioseguridad en la prescripción y transcripción de la terapia medicamentosa endovenosa, b) Relacionar legibilidad en prescripción y transcripción en el Servicio de Neonatología, Temuco 2008-2009, con el propósito de generar un diagnóstico de eventos adversos en la prescripción y transcripción de la terapia medicamentosa endovenosa. Estudio de corte transversal, con muestra probabilística de 125 prescripciones de medicamentos endovenosos. Recolección de información a través de instrumento del American Academy of Pediatrics. Análisis en STATA v10.0. Se consideró: asentimiento ético de jefe Servicio y de la Dirección del Hospital. Conjuntamente, los padres de los neonatos firmaron consentimiento informado. En prescripción 33,6\% no cumple el estándar de seguridad. En escala de acciones incorrectas, categoría "Una acción incorrecta" 20,0\% y 3,2\% "Todas las acciones incorrectas". En transcripción, escala de acciones incorrectas, arroja "Una acción incorrecta" 1,6\% y "Dos acciones incorrectas" $0,8 \%$. En la evaluación del ítem transcripción de fármacos sin indicación, no hubo fármacos transcritos sin indicación. Todas las asociaciones fueron significativas, p: 0,0000. Asegurar la inexistencia de errores en el proceso de prescripción y transcripción de medicamentos debe ser nuestra meta, teniendo en consideración la presencia de factores adversos. Por otra parte, se deben establecer protocolos de bioseguridad, sujetos a evaluación como indicadores de proceso y de resultado, según requieran las políticas en materia de calidad y gestión inmersas en la Reforma en Salud. En este contexto enfermería debe propiciar un rol garante en sus acciones de cuidado. Se espera que la investigación realizada incentive a generar indicadores de resultados y trazadores de calidad, aplicables en los diferentes centros hospitalarios del país.
\end{abstract}

Palabras clave: Bioseguridad, prescripción, transcripción.

\begin{abstract}
The mistakes in the process of medication in your phases, it is a medicine problem, that involves risks for the user, the quality of attention of the services, the professional exercise (fiscal year) and the development of those who are the persons in charge of this process. The objective were: a) To determine the bioseguridad in the prescription and transcription of therapy medicines endovenous, b) Relate legibibility in prescription and transcription in the Service of Neonatology. Hospital Dr. Hernán Henríquez Aravena. Temuco, 2008-2009. Study of transverse court, with sample probabilistic of 125 prescriptions of medicines endovenous. The compilation of the information across instrument of American Academy of Pediatrics validated and adapted to the aims of the study. The analysis in STATA v 10.0. It was considered: Chief's ethical assent Service and of the Direction of the Hospital. Together, the parents of the new born signed informed assent. In Prescription: 33, 6\% does not

\footnotetext{
${ }^{1}$ Proyecto de Investigación No DI08-0069. Financiado por la Dirección de Investigación de la Universidad de La Frontera.

*Enfermera, Doctora en Enfermería, Universidad de La Frontera. Temuco, Chile. E-mail: erivas@ufro.cl

${ }^{* *}$ Enfermera, UCI Neonatal, Hospital Hernán Henríquez Aravena. Temuco, Chile. E-mail: ayrivasl@gmail.com

*** Bioestadístico, Universidad de La Frontera. Temuco, Chile. E-mail: lbustos@ufro.cl
} 
fulfill the safety standard. In scale of incorrect actions, category "An incorrect action" 20, $0 \%$ and 3, $2 \%$ "All the incorrect actions". In Transcription: scale of incorrect actions, throws "An incorrect action" $1,6 \%$ and "Two incorrect actions" $0,8 \%$. In the evaluation of the transcription of medicaments without indication, there were not medicaments transcribed without indication. All the associations were significant, p: 0, 0000. To assure the nonexistence of mistakes in the process of prescription and transcription of medicines must be our goal, taking into account the presence having of adverse factors. On the other hand, we must establish biosecurity protocols, subject to evaluation as indicators of process and outcome, as required by policies and management quality embedded in the Health Reform. In this context, nurses should encourage an guarantor role in their actions carefully. Is expected to encourage research to generate performance indicators and tracers of quality applicable in different hospitals of the country.

Key words: Biosegurity, prescription, transcription.

Fecha recepción: 20/08/09 Fecha aceptación: 22/06/10

\section{INTRODUCCIÓN}

La práctica de medicación es definida como un sistema complejo e interdependiente, en el que intervienen diversos profesionales que comparten un objetivo común, que se traduce en la instalación de ayuda a la salud de los pacientes con calidad, eficacia y seguridad (1). Por Bioseguridad se concibe al conjunto de medidas y disposiciones cuyo objetivo principal es la protección humana (2). Así, el desarrollo del proceso de medicación, por contener variadas fases (prescripción, transcripción, preparación y administración), puede conducir a errores y la falencia en uno de los eslabones altera la continuidad de este proceso, llevando a situaciones de riesgo en la bioseguridad del usuario.

National Coordinating Council for Medication Error Reporting and Prevention (NCCMERP) define los errores de medicación como "cualquier incidente que pueda causar daño al paciente o dé lugar a una utilización inapropiada de los medicamentos, cuando éstos están bajo el control de los profesionales sanitarios o del paciente o consumidor" (3).

Estos incidentes pueden relacionarse con la práctica profesional, con los procedimientos o con los sistemas, incluyendo alteraciones en la prescripción, comunicación, etique- tado, envasado, denominación, preparación, dispensación, distribución, administración, educación, seguimiento y utilización.

A pesar de la importancia creciente de la gestión de riesgos y seguridad del paciente, los datos publicados en el ámbito pediátrico internacional por Muñoz et al. son preocupantes e insuficientes (4), situación que nos motivó a plantearnos la siguiente pregunta de investigación: ¿Cuál es la bioseguridad en la prescripción y transcripción de la terapia medicamentosa endovenosa en el Servicio de Neonatología?

El propósito de la investigación fue generar un diagnóstico de eventos adversos en la prescripción y transcripción de la terapia medicamentosa endovenosa, basándose en la hipótesis de la existencia de errores en la cadena del proceso de medicación.

Los errores de medicación son estudiados mundialmente como errores en la prescripción, transcripción, y administración de fármacos, y varios países están dedicando muchos esfuerzos y dinero para identificarlos y prevenirlos. En nuestro medio no existen indagaciones respecto del tema en general o de alguno de sus componentes. De igual forma no se han encontrado estudios de prevención de errores del proceso, limitándose al estudio de factores indeseados.

Respecto de la magnitud del problema, un estudio español de Campino et al. reportó ta- 
sas de errores de medicación en los procesos de prescripción con un 35,2\% y transcripción 21,3\%. Los errores de prescripción más frecuentes fueron por dosificación $(46,5 \%$ del total de errores). En cuanto al proceso de transcripción, el 30,8\% de los errores se debieron a la ausencia de registro de la vía de administración del fármaco (5).

Las tasas de errores de medicación y de acontecimientos adversos potenciales, entendiéndose como tales prescripciones y transcripciones incorrectas, observados en los diferentes estudios publicados, varían fundamentalmente en función del tipo de paciente estudiado y del método empleado en su detección. Kausal et al. (6) observaron que las tasas de acontecimientos adversos potenciales fueron superiores en neonatos que en otros grupos de edad, con un $20 \%$ frente a $5 \%$ en lactantes, $8 \%$ en preescolares, $12 \%$ en escolares, $11 \%$ en adolescentes y $14 \%$ en adultos. Un subanálisis posterior, realizado por Fortescue et al., comparando las tasas de error y acontecimientos adversos potenciales entre neonatos ingresados en Unidad Cuidados Intensivos Neonatal (UCIN) y en unidades de menor nivel asistencial, reveló que en las UCIN se produce mayor número de errores $(91 \%$ frente a $46 \%$ ocurridos en otros servicios pediátricos) y acontecimientos adversos potenciales $(50 \%$ frente a $9 \%)(7)$.

$\mathrm{Al}$ respecto, un estudio realizado en Brasil por Miasso et al., denominado "The process of preparation and medicine administration: identification of problems to consider improvements and to prevent medication errors" (8), reveló errores en la preparación, relativas a la técnica, preparación anticipada de los medicamentos y fallas en la técnica de comunicación e identificación del paciente en el hospital. Además, estudios de Cohen y Reason consideraron que las causas de estos errores pueden ser relacionadas con factores individuales como la carencia de atención, problemas de memoria, carencias de la formación académica, inexperiencia, pero, tam- bién, con imperfecciones como: problemas en el ambiente (iluminación, interrupciones), carencia en educación (entrenamiento), carencia de profesionales, alteración en la comunicación, problemas en la política y procedimientos o semejantes productos utilizados inadecuadamente en la medicación del paciente (9-10).

Conjuntamente, AEB Hisses analizó los sistemas de medicación en un hospital universitario en Brasil, señaló que los profesionales implicados en cada uno de los procesos descritos deben conocer el sistema desde la medicación y el actuar recíproco de sus componentes, de los cuales depende el resultado final. El profesional debe conocer su rol en la cadena de acciones necesarias a la medicación de un paciente, de modo que su actuar debe ser a conciencia, con responsabilidad y eficacia (11). Al respecto, Leape señala que los profesionales de enfermería son capaces de interceptar alrededor de un $86 \%$ de los errores de medicación, en los procesos de transcripción y sólo el $2 \%$ de los errores es interceptado en la administración de ellos (12). La American Pharmaceutical Association indicó que el uso de la vía electrónica redujo los errores por ilegibilidad en transcripción y prescripción (9).

\section{Enfoque de seguridad y calidad}

En el contexto de prevención de errores, Donabedian (13) hace referencia al aseguramiento de la calidad en los aspectos de índole técnico-científico que se refieren a: oportunidad, seguridad, continuidad, accesibilidad, pertinencia y a la adecuada relación interpersonal, expresada en el respeto a los derechos de los pacientes, sus familias y los adecuados procesos de comunicación, además del uso racional de los recursos de salud (13). En este concepto de seguridad se explicitan los derechos del paciente y la información que debe recibir.

Por seguridad del paciente se entiende "el conjunto de elementos estructurales, proce- 
sos, instrumentos y metodologías basadas en evidencia científicamente probada, que propenden a minimizar el riesgo de sufrir un evento adverso en el proceso de atención de salud o de mitigar sus consecuencias" (14). La seguridad hace parte de la caracterización de calidad de un Servicio de Salud con la oportunidad, accesibilidad, pertinencia y continuidad. Sin embargo, alteraciones en estos conceptos pueden producir lesiones, denominadas eventos adversos, resultantes de la intervención de los Servicios de Salud, produciendo disfunción que puede ser medida. En este caso se podría decir que es el daño hacia el paciente ocasionado por el contacto con un Sistema de Salud.

Existen múltiples formas de clasificar el evento adverso, una de las más útiles respecto de la definición de responsabilidad ética, penal, civil y administrativa, es aquella que divide en evento adverso prevenible y no prevenible. El evento adverso no prevenible es la complicación que no puede ser evitada dado el estado actual del conocimiento; al contrario, el evento adverso prevenible es el mal resultado de la atención que puede ser evitado con el estado del conocimiento.

$\mathrm{Al}$ respecto, la herramienta parece ser la gestión del riesgos que se traduce en "el análisis de los procesos de atención, de su estructura y resultados que permite la prevención de los eventos inesperados o el manejo oportuno de sus consecuencias para el paciente, el profesional de la salud, la instituciones prestadoras de salud y el sistema”.

En el reporte de Campino et al., "Errar es humano: construyendo un sistema de salud más seguro" (To err is human: Building a safer health system) (5), producido por The Institute of Medicine en 1999, evidenció la carga sobre la mortalidad general de los errores del sistema de salud en los Estados Unidos, siendo éstos la tercera causa de muerte, después de la enfermedad cardiaca y el cáncer. El número de errores potencialmente prevenibles cada año variaba entre
44.000 y 98.000 muertes, superando incluso los fallecimientos por accidentes de vehículos automotores, cáncer mamario y SIDA.

Según Moraima, cerca del $70 \%$ de los eventos adversos son prevenibles. Los errores pueden evitarse si se diseñan sistemas que muestren dificultades para equivocarse y facilidades para hacer las cosas bien (15).

Para Gómez y Espinoza, el evento adverso no sólo tiene impacto en el paciente y su familia por las lesiones físicas o el daño psicológico que produce, sino también en los profesionales de la salud responsables de estas situaciones (estrés postraumático, depresión, inicio de ejercicio profesional a la defensiva, costos en el patrimonio y el buen nombre), en el sistema de salud por los costos que representan la atención de los eventos adversos (entre 17 y 29 billones de dólares al año), y en la sociedad por la pérdida en la credibilidad en la calidad de los servicios cuando estas situaciones emergen al público general (16).

La Organización Mundial de la Salud (OMS) (17), en el año 2002, emitió una resolución para mejorar la seguridad de los pacientes, que se concretó en el año 2004 con el establecimiento de la Alianza Mundial por la Seguridad de los Pacientes, como una de las prioridades de Salud Pública. Definió 21 indicadores para vigilar y evaluar el desempeño de los Sistemas de Salud en materia de seguridad del paciente, los cuales fueron: infecciones nosocomiales, eventos centinelas (eventos adversos relacionados con el equipo médico y errores de medicación), complicaciones quirúrgicas y posquirúrgicas, complicaciones obstétricas y otros eventos adversos.

\section{Aspecto ético legal}

En materia de bioseguridad, se hace indispensable tener un referente ético, al respecto Savater (18) lo define "como una propuesta de reflexión sobre la propia libertad que consiste en buscar lo mejor y ayudar a cumplir 
el ideal del hombre". En un dilema ético la materia de decisión es de carácter moral, es decir, cómo garantizar el respeto a los principios fundamentales del accionar. En este contexto al referirse a bioseguridad se hace alusión a propiciar los cuidados y tratamientos de manera confiable, considerando los principios señalados.

Kidder (19) aclara que un dilema ético no consiste en tener que hacer una elección entre lo correcto y lo incorrecto, sino en elegir entre dos cosas que son correctas, pero desde perspectivas distintas. El personal responsable de la terapia medicamentosa debe tener la capacidad para prevenir problemas o reconocer rápidamente los dilemas éticos, analizar, argumentar, y encontrar las posibles soluciones a éstos. De esta forma los problemas de seguridad en la atención de los pacientes se deben valorar a la luz de "las circunstancias de tiempo, modo y lugar que rodearon los hechos, y las precauciones que frente al mismo hubiera aplicado un profesional prudente y diligente" (13).

\section{Objetivos generales}

- Determinar la bioseguridad en la prescripción y transcripción de medicamentos endovenosos en el Servicio de Neonatología. Temuco 2008-2009.

- Relacionar la legibilidad en prescripción y transcripción de la terapia medicamentosa endovenosa en el Servicio de Neonatología. Temuco 2008-2009.

\section{Objetivos específicos}

- Identificar el estado de la prescripción medicamentosa endovenosa: dosis, vía y frecuencia de administración del medicamento.

- Establecer el estado de la transcripción medicamentosa endovenosa.

\section{MATERIAL Y MÉTODO}

Estudio de corte transversal que incorporó una muestra probabilística de 125 prescripciones de medicamentos endovenosos, estimada con una confianza de $95 \%, p=0,352$ y $q=0,648$, error muestral 7,5\%, en una población de 1.200 niños. La recolección de la información se realizó a través del instrumento del American Academy of Pediatrics, validado y adecuado a los objetivos del estudio. Las prescripciones se obtuvieron de las fichas clínicas y se utilizó pauta de observación con criterios de cumplimiento. Para controlar la calidad de los registros se realizó análisis de valores extremos y se siguieron los estándares de la American Academy of Pediatrics y de la American Society of Health-System Pharmacist utilizados universalmente y ampliamente validados, conjuntamente se trabajó con químico farmacéutico. En los registros se evaluó: En indicación: legibilidad, dosificación, vía e intervalos de administración. En transcripción: legibilidad, errores por omisión, no prescripción.

Análisis realizado en STATA v10.0. En un primer momento se efectuó análisis descriptivo con distribución porcentual de frecuencias y, en un segundo momento, análisis inferencial, estableciendo asociaciones a través de pruebas estadísticas (Chi cuadrado y Pearson).

Se consideró: asentimiento ético de jefe Servicio y de la Dirección del Hospital. Conjuntamente, los padres de los neonatos firmaron consentimiento informado.

\section{RESULTADOS}

En bioseguridad de la prescripción se observó que un 33,6\% no cumple el estándar de seguridad, siendo la categoría "No señala vía de administración", quien presentó el mayor porcentaje (Tabla 1). 
En prescripción de medicamentos, evaluada en escala de acciones incorrectas, la categoría "Una acción incorrecta" 20,0\% y $3,2 \%$ "Todas las acciones incorrectas" (Tabla 2).

En transcripción de medicamentos, evaluada en escala de acciones incorrectas, la categoría "Una acción incorrecta" reunió 1,6\% y "Dos acciones incorrectas" 0,8\% (Tabla 3).

En la relación de prescripción y transcripción de medicamentos se encontró 28 trans- cripciones no legibles derivadas de prescripciones legibles. Por otra parte, se observó una falta de transcripción derivada de prescripción legible. No hubo transcripción de medicamentos sin indicación (Tabla 4).

Todas las asociaciones en relación a prescripción según legibilidad de la transcripción, transcripción de todos los fármacos indicados y transcripción de fármacos sin indicación, fueron significativas, p: 0,0000 (Tabla 4).

Tabla 1. Prescripción y transcripción de medicamentos. Servicio Neonatología. Temuco, 2008-2009.

\begin{tabular}{|lrr|}
\hline & f & $\%$ \\
\hline Prescripción & & \\
No Señala dosis & 9 & 7,2 \\
No señala vía de administración & 20 & 16,0 \\
No señala frecuencia de administración & 13 & 10,4 \\
\hline Transcripción & & \\
Fármacos indicados, sin transcripción & 1 & 0,8 \\
Fármacos no indicados transcritos & 1 & 0,8 \\
\hline
\end{tabular}

Fuente: Proyecto DI UFRO. Bioseguridad en la terapia medicamentosa endovenosa. Servicios Pediátricos. Temuco, 2008-2009.

Tabla 2. Prescripción de medicamentos en relación a escala de acciones incorrectas. Servicio Neonatología. Temuco, 2008-2009.

\begin{tabular}{|lrc|}
\hline & f & \% \\
\hline Todas correctas & 84 & 67,2 \\
Una acción incorrecta & 25 & 20,0 \\
Dos acciones incorrectas & 7 & 5,6 \\
Tres acciones incorrectas & 5 & 4,0 \\
Todas las acciones incorrectas & 4 & 3,2 \\
\hline
\end{tabular}

Fuente: Proyecto DI UFRO. Bioseguridad en la terapia medicamentosa endovenosa. Servicios Pediátricos. Temuco, 2008-2009. 
Tabla 3. Transcripción de medicamentos en relación a escala de acciones incorrectas. Servicio Neonatología. Temuco, 2008-2009.

\begin{tabular}{|lcc|}
\hline & f & $\%$ \\
\hline Todas correctas & 122 & 97,6 \\
Una acción incorrecta & 2 & 1,6 \\
Dos acciones incorrectas & 1 & 0,8 \\
Todas las acciones incorrectas & 0 & 0,0 \\
\hline
\end{tabular}

Fuente: Proyecto DI UFRO. Bioseguridad en la terapia medicamentosa endovenosa. Servicios Pediátricos. Temuco, 2008-2009.

Tabla 4. Prescripción según legibilidad de la transcripción, transcripción de todos los fármacos indicados y transcripción de fármacos sin indicación. Servicio de Neonatología. Temuco, 2008-2009.

\begin{tabular}{|l|c|c|c|}
\hline & Prescripción legible & Prescripción no legible & p \\
\hline Transcripción legible & 95 & 2 & 0,0000 \\
Transcripción no legible & 28 & 0 & 0,0000 \\
\hline Todos los fármacos transcritos & 96 & 28 & 0 \\
No todos los fármacos transcritos & 1 & 1 & 0,0000 \\
\hline Transcripción de fármacos sin indicación & 96 & 28 & \\
No transcripción de fármacos sin indicación & 0 & 0 & \\
\hline
\end{tabular}

Fuente: Proyecto DI UFRO. Bioseguridad en la terapia medicamentosa endovenosa. Servicios Pediátricos. Temuco, 2008-2009.

\section{DISCUSIÓN}

El proceso de medicación en los pacientes es un acto complejo, dinámico y expuesto a riesgos, por lo que se puede incurrir en faltas y omisiones, afectando la seguridad de quienes reciben la medicación. Por tanto, se deben revisar continuamente los indicadores del proceso de prescripción y transcripción de fármacos.

En nuestro estudio, en el proceso de prescripción de fármacos encontramos elevado porcentaje $(33,6 \%)$ de omisión de dosis, vía y frecuencia de administración de medicamentos.
Por otra parte, el proceso de prescripción mostró importante índice $(32,8 \%)$ de acciones incorrectas respecto a las categorías: transcripción legible, dosis, vía y frecuencia de administración de fármacos. Existiendo $20,0 \%$ de estas categorías con al menos una acción incorrecta y $3,2 \%$ con todas las acciones incorrectas.

En el ámbito de la relación entre prescripción versus transcripción no legible, transcripción de todos los fármacos indicados y transcripción de fármacos sin indicación se encontró asociación significativa.

El proceso de prescripción por sí solo es la base y eslabón fundamental en la cadena de medicación, ya que da inicio a una serie 
de acciones que deben ser ejecutadas por el equipo de salud.

Por tanto una prescripción correcta (legible, que describa dosis, vía y frecuencia de administración) certifica una correcta comprensión de quien debe transcribir el fármaco y de esta forma asegura que el paciente reciba lo que por indicación le corresponde a su tratamiento.

Sin duda, la legibilidad en las prescripciones y transcripciones es una cualidad que está en directa relación con la seguridad del paciente, por lo que los porcentajes de acciones incorrectas $\mathrm{u}$ omitidas arrojados en la investigación conducen a faltas en las estrategias de calidad e incumplimiento de los objetivos sanitarios en materia de bioseguridad de administración de fármacos. Se debe contar con estrategias en la medicación, para evitar acontecimientos adversos, siendo muchos de ellos prevenibles (20). Como mecanismo de control se sugiere que las organizaciones desarrollen una cultura de seguridad dirigida hacia el paciente, con el objetivo de detectar acciones incorrectas diseñando estrategias globales para disminuir su incidencia y cuantificar la magnitud del cambio alcanzado (5).

En las unidades corresponde facilitar la formación del personal insistiendo en la importancia de la prescripción, transcripción y registros correctos, estableciendo programas de declaración anónima, voluntaria y no punitiva de errores (21), programas informáticos que faciliten los cálculos de dosis y diluciones necesarias de acuerdo a las características del paciente (22), establecer sistemas de seguridad (doble firma, restricciones según el nivel de experiencia) para las prescripciones o preparaciones realizando controles de seguridad de los pacientes (23), e incluir a las redes de apoyo (farmacia) en las peculiaridades y necesidades específicas de los niños y de los recién nacidos.

Pero estas medidas, la mayor parte de ellas reactivas, aunque importantes, son insuficientes (24). Se deben implantar programas a nivel hospitalario que analicen de un modo proactivo y multidisciplinario las causas de los errores desde un punto de vista conceptual y en los procedimientos concretos, ya que muchos de los errores están relacionados más con fallos en los procesos y con defectos de comunicación entre los profesionales que con incompetencia de los mismos (25).

En el aspecto ético: la seguridad del paciente, dentro de una estrategia de calidad total, es un objetivo fundamental en todo sistema sanitario y unidad asistencial. Errar es humano (26), por lo que resulta dificultoso conseguir la ausencia total de faltas. Debido a lo anterior, la meta en gestión en salud debe apuntar a minimizar el riesgo de acción-error por parte de los profesionales de salud, teniendo en cuenta los factores externos que pueden propiciar las fallas. El primer paso que se debe tener presente a la hora de implementar un sistema de gestión de riesgos es la identificación de errores (27). Dentro de éstos encontramos la revisión de historias clínicas, la notificación voluntaria, auditorías o encuestas de satisfacción. Cada uno de estos métodos tiene sus ventajas e inconvenientes; así la revisión de historias clínicas es un sistema exhaustivo de búsqueda de errores pero muy costoso. Por otra parte, en la notificación voluntaria de incidentes o de casi-incidentes (28), no todas las personas están dispuestas a constatar los errores, ya sea por falta de tiempo, por miedo a sanciones, discriminación por parte de sus pares o simplemente por temor a la pérdida de la confianza en ellos.

Se ha demostrado que las intervenciones para mejorar la educación del personal y la conciencia de los errores son eficaces en reducir riesgos, aunque sea difícil cuantificar la proporción de la disminución de éstos. La inexperiencia es un factor de riesgo particular para errores de medicación, lo cual es apoyado por hallazgos de que el personal nuevo comete errores con mayor probabilidad. Esto sumado, al hecho que las prescripciones a menudo son escritas por médicos 
jóvenes que pueden no estar familiarizados con la medicina (29).

Estudios apuntan al cambio de mentalidad en las estrategias de búsqueda del error y señalan que éstas deben basarse en dos puntos fundamentales: un cambio en la cultura del buscar culpables y que los errores deben ser considerados fallas del sistema, debiendo ser notificados con el fin de mejorar la calidad asistencial. El objetivo final debe ser la mejora de los procedimientos de trabajo para evitar que vuelvan a suceder (5).

Se pueden establecer patrones o estándares de comportamiento que prevengan acontecimientos indeseables, conversar con el personal, generando confianza de notificar las alteraciones, mentalizando en que el desarrollo de una buena práctica de salud en el cuidado de los pacientes debe contar con veracidad, prevención y de la empatía.

En el aspecto legal: los registros adquieren destacada importancia, Ruiz (30) los define como "el soporte documental donde queda recogida la totalidad de la información respecto de la actividad del profesional de salud, referente a una persona concreta, su valoración, tratamiento recibido y evolución". Plantea que la consignación de los datos se fundamenta en la responsabilidad moral, en el respeto a los derechos de los pacientes y la responsabilidad profesional.

Finalmente, estudios enfatizan que se debe promover la notificación voluntaria de los errores, obteniendo un relato correcto como forma de registro, para el servicio y como protección legal en caso de un proceso ético o jurídico (31).

Asegurar la inexistencia de errores en el proceso de prescripción y transcripción de medicamentos debe ser nuestra meta, teniendo en consideración los factores adversos que intervienen en él. Sin embargo, creemos que se deben establecer patrones de bioseguridad sobre los cuales cada institución en salud debe ejecutar sus acciones, sujetos a evaluación y modificación, según requieran las políticas en materia de calidad y gestión inmersas en la Reforma en Salud.

Se espera que la investigación realizada incentive a obtener indicadores de calidad tanto de proceso como de resultado, generando estándares aplicables en cualquier centro hospitalario del país.

\section{REFERENCIAS}

1. Nadzan DM. The System Approach you the Medication Use. In: Cousins DM. Medication Use: The System Approach You the Reducing Errors. Oakbrook Terrace (IL): Joint Commission. 1998 :518.

2. Comité Nacional de Biotecnología. Manual de Normas de Bioseguridad. CONYCIT; 1994 . Hallado en: www.fondecyt. cl [Acceso en septiembre 2009].

3. National Coordinating Council for Medication Error Reporting and Prevention (NCCMERP). Hallado en: http.//www. nccmerp.org/aboutMedErrors.htlm [Acceso en septiembre 2009].

4. Muñoz M, Pallás C, De la Cruz J, López M, Moral A, Balaustegui A. Errores en las prescripciones médicas en una unidad neonatal. An Esp Pediatr. 2001;55: 53540.

5. Campino A, López M, García M, López de Heredia I, Valls i Soler A. Errores en la prescripción y transcripción de medicación en una unidad neonatal. Unidad Neonatal. Servicio de Pediatría. Hospital de Cruces. Baracaldo. Bilbao. España. Anales de Pediatría Barcelona. 2006; 64:330-5. Hallado en: http://www.doyma.es/revistas/ctl_servlet?_f $=7064 \&$ ip $=146.83 .205 .13 \&$ articuloid $=13086520$ [acceso en septiembre 2009].

6. Kausal R, Bates DW, Landrigan C, Mckenna K, Clapp MD, Federico F, et al. Medication errors and adverse drug events in pediatric inpatients. JAMA. 2001; 285:2114-20. 
7. Fortescue EB, Kaushal R, Landrigan CP, McKenna KJ, Clapp MD, Federico F, et al. Prioritizing strategies for preventing medication errors and adverse drug events in pediatric inpatients. Pediatrics, 2003; 111:722-9.

8. Miasso AI, Silva AEBC, Cassiani SHB, Grou CR, de Oliveira RC, Fakih FT. The process of preparation and medicine administration: identification of problems to consider improvements and to prevent medication errors Rev. Latin-Am. Enfermagem. 2006; 14: 354-63.

9. Cohen MR. Preventing Medications Errors related you the Prescribing. In: Cohen MR. Medication Errors Causes, Prevention, and Risk Management. Washington: American Pharmaceutical Association. 2000; 8.23.

10. Reason J. Human Error. Journal of Perinatal \& Neonatal Nursing. 2002; 9.

11. AEB Hisses. Analysis of the medication system of a university hospital of the state of Goiã's. School of Nursing, 2003.

12. Leape LL, You beat DW, Cullen DJ, Cooper J, Demonaco HJ, Gallivan T, et al. System analisys of opposite's drug events. JAMA, 1995; 274(1):35-43.

13. Donabedian A. La calidad de atención Médica. México DF: Prensa Médica Mexicana, 1984: 95-151.

14. Respuesta del Ministerio de la Protección Social. Revista de Medicina. Hallado en: http://www.encolombia.com/medicina/ academedicina/Academ300208/Respuestadelministerio1.htm [acceso en julio 2008].

15. Moraima WD, Leiva D., Bujardón A., Bandera T., Vera JE. Bioética. Una mirada hacia la enfermería. Revista Cubana de Enfermería. 1999; 15(2):85-9.

16. Gómez Ana, Espinoza A. Ethical Dilemmas Facing the Patient's Safety. Care Means Ponder. Colombia. 2006; 6 (1):5467.

17. OMS. Uso racional de medicamentos.
Informe de la Conferencia de Expertos. Nairobi; 1985.

18. Savater, F. El valor de elegir. Editorial Ariel. Barcelona, 2003.

19. Kidder, R. Ethical Leadership. Editorial Amazon. EEUU, 1995.

20. Best F. Recommendations for the practical ones of the medication - coalition for the prevention of medical errors of Massachusetts en Cassini HB, Ureta J. The security of the patients in the use of the medication. Medical arts. 2004.

21. Suresh G, Horbar JD, Plsek P, Gray J, Edwards $\mathrm{WH}$, Shiono PH, et al. Voluntary anonymous reporting of medical errors for neonatal intensive care. Pediatrics, 2004; 113:1609-18.

22. Kaushal R, Barker KN, Bates DW. How can information technology improve patient safety and reduce medication errors in chidren's health care? Arch Pediatr Adolesc Med. 2001;155:1002-7.

23. Ursprung R, Gray JE, Edwards WH, Horbar JD, Nickerson J, Plsek P, et al. Real time patient safety audits: Improving safety every day. Qual Saf Health Care, 2005; 14:284-9.

24. Resar R. Will, ideas, and execution: Their role in reducing adverse medication events. J Pediatr. 2005; 147:727-8.

25. Donchin Y, Gopher D, Olin M, Badihi Y, Biesky M, Sprung CL, et al. A look into the nature and causes of human errors in the intensive care unit. Qual Saf Health Care. 2003; 12: 143-8.

26. Committee on Quality of Health Care in America. Errors in health care: A leading cause of death and injury en Kohn L, Corrigan J, Donaldson M, editors. To err is human: Building a safer health system. Institute of Medicine. Washington: National Academy Press. 1999; 26-48.

27. Edwards WH. Patient safety in the neonatal intensive care unit. Clin Perinatol. 2005; 32:97-106.

28. Taylor JA, Brownstein D, Christakis A, Blackburn S, Strandjord TP, Klein EJ, et 
al. Use of incident reports by physicians and nurses to document medical errors in pediatric patients. Pediatrics. 2004; 114:729-35.

29. Simpson, J H y cols. Reduciendo errores de medicamentos en Unidad de Cuidado Intensivo Neonatal. Pediatric Department, Princess Royal Maternity Hospital, Glasgow, Scotland, UK Archives of disease in Childhood Fetal and Neonatal,
2004; 89(6) :480-482.

30. Ruiz A. Calidad en los Registros de Enfermería, 2005. Hallado en: http://www. fac.org.ar/ccvc/llave/c028/ruizhont.php [acceso en mayo 2009].

31. Carvalho V, Cassiani H. Medication errors and consequences for nursing professionals and clients. Rev. LatinoAm. Enfermagem. 2002; 10(4): 523-529. 